\title{
Telomere length in circulating leukocytes is associated with lung function and disease
}

\author{
Eva Albrecht ${ }^{1}$, Elina Sillanpää ${ }^{1}$, Stefan Karrasch ${ }^{1}$, Alexessander Couto Alves ${ }^{1}$, \\ Veryan Codd, liris Hovatta, Jessica L. Buxton, Christopher P. Nelson, \\ Linda Broer, Sara Hägg, Massimo Mangino, Gonneke Willemsen, Ida Surakka, \\ Manuel A.R. Ferreira, Najaf Amin, Ben A. Oostra, Heli M. Bäckmand, \\ Markku Peltonen, Seppo Sarna, Taina Rantanen, Sarianna Sipilä, \\ Tellervo Korhonen, Pamela A.F. Madden, Christian Gieger, Rudolf A. Jörres, \\ Joachim Heinrich, Jürgen Behr, Rudolf M. Huber, Annette Peters, \\ Konstantin Strauch, H. Erich Wichmann, Melanie Waldenberger, \\ Alexandra I.F. Blakemore, Eco J.C. de Geus, Dale R. Nyholt, \\ Anjali K. Henders, Päivi L. Piirilä, Aila Rissanen, Patrik K.E. Magnusson, \\ Ana Viñuela, Kirsi H. Pietiläinen, Nicholas G. Martin, Nancy L. Pedersen, \\ Dorret I. Boomsma, Tim D. Spector, Cornelia M. van Duijn, Jaakko Kaprio, \\ Nilesh J. Samani, Marjo-Riitta Jarvelin ${ }^{1}$ and Holger Schulz ${ }^{1}$
}

Affiliations: For a list of the authors' affiliations see the Acknowledgements section, 'These authors contributed equally to this work.

Correspondence: H. Schulz, Helmholtz Zentrum München, German Research Center for Environmental Health, Institute of Epidemiology I, Ingolstädter Landstraße 1, 85764 Neuherberg, Germany. E-mail: schulzahelmholtz-muenchen.de

ABSTRACT Several clinical studies suggest the involvement of premature ageing processes in chronic obstructive pulmonary disease (COPD). Using an epidemiological approach, we studied whether accelerated ageing indicated by telomere length, a marker of biological age, is associated with COPD and asthma, and whether intrinsic age-related processes contribute to the interindividual variability of lung function.

Our meta-analysis of 14 studies included 934 COPD cases with 15846 controls defined according to the Global Lungs Initiative (GLI) criteria (or 1189 COPD cases according to the Global Initiative for Chronic Obstructive Lung Disease (GOLD) criteria), 2834 asthma cases with 28195 controls, and spirometric parameters (forced expiratory volume in $1 \mathrm{~s}$ (FEV1), forced vital capacity (FVC) and FEV1/FVC) of 12595 individuals. Associations with telomere length were tested by linear regression, adjusting for age, sex and smoking status.

We observed negative associations between telomere length and asthma $(\beta=-0.0452, p=0.024)$ as well as $\operatorname{COPD}(\beta=-0.0982, p=0.001)$, with associations being stronger and more significant when using GLI criteria than those of GOLD. In both diseases, effects were stronger in females than males. The investigation of spirometric indices showed positive associations between telomere length and FEV1 $\left(\mathrm{p}=1.07 \times 10^{-7}\right)$, FVC $\left(p=2.07 \times 10^{-5}\right)$, and FEV1/FVC $\left(p=5.27 \times 10^{-3}\right)$. The effect was somewhat weaker in apparently healthy subjects than in COPD or asthma patients.

Our results provide indirect evidence for the hypothesis that cellular senescence may contribute to the pathogenesis of COPD and asthma, and that lung function may reflect biological ageing primarily due to intrinsic processes, which are likely to be aggravated in lung diseases.

@ERSpublications

Telomere length is decreased in asthma and COPD patients and positively associated with spirometric indices http://ow.ly/qOLFa 


\section{Introduction}

An increasing life expectancy is associated with an increased susceptibility for prevailing chronic diseases, such as cardiovascular and neurological diseases, type 2 diabetes and cancer, suggesting that common, agerelated processes are involved in these diseases [1-4]. The lung is known to undergo age-related processes associated with a continuous decline in lung function $[5,6]$. Chronic obstructive pulmonary disease (COPD), lung cancer and idiopathic pulmonary fibrosis are lung diseases with an increase in incidence by age. In COPD, chronic inflammation and oxidative stress are among the generally accepted pathophysiological mechanisms but, as discussed for other degenerative diseases, accelerated ageing resulting in premature cell senescence may contribute to the pathobiology [7-9]. Although clinical and epidemiological studies provide some evidence for this hypothesis, the data base is still limited [8], so our study aimed to provide further clues on this.

Telomeres, made up of the simple repetitive sequence TTAGGG in humans [10], are specialised DNA structures that, in concert with telomere-specific proteins, protect the ends of chromosomes and maintain the integrity of the genome [3]. They progressively shorten with each cell division [11] and, after reaching a critical length, either cellular senescence or apoptosis is induced [3]. Telomeres are therefore considered a marker of biological age. Their length is affected by various factors, such as chronic inflammation or oxidative stress [3], but has also been shown to have a heritable component [12]. Recent data suggest that shorter telomere length is associated with an increased risk of total and cancer mortality in COPD patients [13]. Shortened telomeres were found in pulmonary vascular endothelial cells, alveolar epithelial cells and circulating leukocytes of COPD patients [14-18], supporting the notion of accelerated ageing in COPD. RODE et al. [18] reported multivariable-adjusted odds ratios of 1.15 (95\% CI 1.06-1.25) for shortest versus longest telomere quartiles for COPD. Smoking, a major cause of COPD, is associated with different signs of premature ageing, which is particularly obvious in the skin [8], suggesting that environmental exposure may play an important role in triggering processes related to biological ageing.

Since the lung is continuously exposed to environmental hazards, lung function per se may be a surrogate marker for biological age in light of the large interindividual variability observed [19]. Only a few casecontrol studies $[14,20]$ have investigated the relationship between telomere length and spirometric indices. One large population-based study was recently published by RoDE et al. [18] reporting a modest correlation between telomere length and lung function in terms of the spirometric indices forced expiratory volume in $1 \mathrm{~s}$ (FEV1), forced vital capacity (FVC) and their ratio, FEV1/FVC.

In a meta-analysis of 14 European studies embedded in the ENGAGE (European Network for Genetic and Genomic Epidemiology) consortium, we studied the relationship between telomere length, lung function and lung disease to address the question of whether accelerated ageing, indicated by telomere length, may be involved in the pathogenesis of COPD and asthma, and whether intrinsic age-related processes may contribute to the interindividual variability of lung function. For this purpose, we explored the association between circulating leukocyte telomere length and COPD or asthma. Although these are different obstructive disease entities, they share chronic inflammation as a hallmark of disease, which may result in accelerated ageing and telomere shortening. We further investigated the association between telomere length and lung function using the spirometric indices FEV1, FVC and FEV1/FVC.

\section{Methods}

\section{Study populations}

This meta-analysis comprises data from 14 European studies, with details provided in the online supplementary material. In the analysis of telomere length and COPD, we combined data of nine studies (EX-ATHLETES, the Finnish Twin Study on Ageing (FITSA), Cooperative Health Research in the Region Augsburg (KORA) Age, KORA F3, KORA F4, Nicotine Addiction Genetics Finland (NAG-FIN), Northern Finland Birth Cohort Study (NFBC) 1966, TwinGene and TwinsUK), totalling 934 cases and 15846 controls (table 1).

COPD was defined as an FEV1/FVC z-score $<-1.6445$ ( $5 \%$ lower limit of normal (LLN) according to the Global Lungs Initiative (GLI) [19]) if spirometry was available (see later) or based on physician diagnosis. In a sensitivity analysis, we replaced the GLI criteria by the Global Initiative for Chronic Obstructive Lung Disease $(\mathrm{GOLD})$ criteria $(\mathrm{FEV} 1 / \mathrm{FVC}<0.70)$ [21] to define COPD. The analysis of telomere length in asthma comprised 13 studies (ERF (Erasmus Rucphen Family), EX-ATHLETES, FITSA, GRAPHIC, KORA Age,

This article has supplementary material available from www.erj.ersjournals.com

Received: March 142013 | Accepted after revision: Oct 302013 | First published online: Dec 052013

Conflict of interest: Disclosures can be found alongside the online version of this article at www.erj.ersjournals.com 
TABLE 1 Characteristics of studies in chronic obstructive pulmonary disease (COPD) analysis defined by Global Lungs Initiative criteria

\begin{tabular}{|c|c|c|c|c|c|c|c|c|}
\hline \multirow[t]{2}{*}{ Study } & \multirow[t]{2}{*}{ Subjects } & \multirow[t]{2}{*}{ COPD cases } & \multirow[t]{2}{*}{ Females } & \multirow{2}{*}{$\begin{array}{c}\text { Smoking status } \\
\text { never/former/ } \\
\text { current }\end{array}$} & \multicolumn{4}{|c|}{ Age years } \\
\hline & & & & & Overall & Cases & Controls & Difference \\
\hline EX-ATHLETES & 577 & $43(7.5)$ & $0(0)$ & $361 / 177 / 39$ & $72.68 \pm 5.92$ & $74.63 \pm 6.97$ & $72.52 \pm 5.81$ & 2.11 \\
\hline FITSA & 386 & 22 (5.7) & $386(100)$ & $333 / 31 / 21$ & $68.45 \pm 3.38$ & $69.27 \pm 3.60$ & $68.40 \pm 3.36$ & 0.87 \\
\hline KORA Age & 905 & 54 (6.0) & 451 (49.8) & $510 / 351 / 44$ & $75.67 \pm 6.34$ & $75.02 \pm 6.28$ & $75.71 \pm 6.35$ & -0.69 \\
\hline NAG-FIN & 1718 & 126 (7.3) & $782(45.5)$ & $204 / 804 / 710$ & $55.89 \pm 7.63$ & $58.40 \pm 9.40$ & $55.69 \pm 7.44$ & 2.71 \\
\hline NFBC 1966 & 4984 & 147 (2.9) & 2594 (52.0) & 2479/592/1913 & $31.0 \pm 0.2$ & $31.0 \pm 0.2$ & $31.0 \pm 0.2$ & 0 \\
\hline TwinGene & 597 & $52(8.7)$ & 597 (100) & $358 / 239^{\#}$ & $71.72 \pm 5.89$ & $72.50 \pm 5.31$ & $71.65 \pm 5.95$ & 0.85 \\
\hline TwinsUK & 3935 & $211(5.4)$ & 3687 (93.7) & $2760 / 743 / 432$ & $51.17 \pm 13.12$ & $53.34 \pm 13.06$ & $51.02 \pm 13.14$ & 2.32 \\
\hline Total & 16780 & $934(5.6)$ & 10435 (62.2) & & & & & $1.71^{\top}$ \\
\hline
\end{tabular}

Data are presented as $\mathrm{n}, \mathrm{n}(\%)$ or mean \pm SD, unless otherwise stated. FITSA: Finnish Twin Study on Ageing; KORA: Cooperative Health Research in the Region Augsburg; NAG-FIN: Nicotine Addiction Genetics Finland; NFBC: Northern Finland Birth Cohort Study. ${ }^{\#}$ : current smoking no/yes;

$\because$ : mean age difference between cases and controls, weighted by total sample size.

KORA F3, KORA F4, NAG-FIN, NFBC 1966, NTR (Netherlands Twin Register), QIMR (Queensland Institute of Medical Research) adolescent study, TwinGene, and TwinsUK), with 2834 cases and 28195 controls (table 2). Asthma was defined by physician diagnosis, by self-report in questionnaires or by asthma-specific medication intake. Spirometric measurements (FEV1, FVC and FEV1/FVC) were available for 12595 individuals from seven studies (FITSA, KORA Age, KORA F3, KORA F4, NFBC 1966, TwinFat and TwinsUK). Spirometry was performed according to international standards [22, 23]. Relative telomere length was determined by quantitative PCR [24] in all studies. Measurement details by study are provided in the online supplementary data. Local ethics committees approved all studies and all participants had given their written informed consent.

TABLE 2 Characteristics of studies in asthma analysis

\begin{tabular}{|c|c|c|c|c|c|c|c|c|}
\hline \multirow[t]{2}{*}{ Study } & \multirow[t]{2}{*}{ Subjects } & \multirow[t]{2}{*}{ COPD cases } & \multirow[t]{2}{*}{ Females } & \multirow{2}{*}{$\begin{array}{c}\text { Smoking status } \\
\text { never/former/ } \\
\text { current }\end{array}$} & \multicolumn{4}{|c|}{ Age years } \\
\hline & & & & & Overall & Cases & Controls & Difference \\
\hline ERF & 2580 & $41(1.6)$ & 1468 (56.9) & $981 / 691 / 908$ & $49.80 \pm 15.67$ & $51.14 \pm 13.35$ & $49.78 \pm 15.71$ & 1.36 \\
\hline EX-ATHLETES & 577 & $44(7.6)$ & $0(0)$ & $361 / 177 / 39$ & $72.68 \pm 5.92$ & $73.23 \pm 5.98$ & $72.63 \pm 5.92$ & 0.60 \\
\hline FITSA & 423 & $33(7.8)$ & $423(100)$ & $369 / 32 / 21$ & $68.61 \pm 3.41$ & $68.27 \pm 3.48$ & $68.64 \pm 3.41$ & -0.37 \\
\hline KORA F3 & 2532 & $191(7.5)$ & 1314 (51.9) & $1133 / 863 / 536$ & $53.62 \pm 10.80$ & $55.77 \pm 9.85$ & $53.44 \pm 10.86$ & 2.33 \\
\hline KORA F4 & 2797 & 231 (8.3) & $1477(52.8)$ & $1228 / 1045 / 524$ & $54.90 \pm 12.85$ & $54.15 \pm 12.75$ & $54.97 \pm 12.85$ & -0.82 \\
\hline NAG-FIN & 1718 & $97(5.6)$ & $782(45.5)$ & 204/804/710 & $55.89 \pm 7.63$ & $59.72 \pm 9.07$ & $55.67 \pm 7.48$ & 4.05 \\
\hline NFBC 1966 & 4843 & $434(9.0)$ & $2516(52.0)$ & $2463 / 590 / 1790$ & $31.0 \pm 0.2$ & $31.0 \pm 0.2$ & $31.0 \pm 0.2$ & 0 \\
\hline NTR & 7529 & 906 (12.0) & 4688 (62.3) & $3734 / 2213 / 1582$ & $42.50 \pm 15.29$ & $41.51 \pm 14.80$ & $42.64 \pm 15.35$ & -1.13 \\
\hline QIMR & 315 & 149 (47.3) & $161(51.1)$ & NA & $20.94 \pm 5.48$ & $20.60 \pm 5.40$ & $21.25 \pm 5.56$ & -0.65 \\
\hline
\end{tabular}

Data are presented as $n, n(\%)$ or mean \pm SD, unless otherwise stated. ERF: Erasmus Rucphen Family; FITSA: Finnish Twin Study on Ageing; KORA: Cooperative Health Research in the Region Augsburg; NAG-FIN: Nicotine Addiction Genetics Finland; NFBC: Northern Finland Birth Cohort Study; NTR: Netherlands Twin Register; QIMR: Queensland Institute of Medical Research; NA: not available. " : current smoking no/yes; ": mean age difference between cases and controls, weighted by total sample size. 


\section{Statistical analysis}

Associations of telomere length with COPD, asthma, FEV1, FVC and FEV1/FVC were tested by linear regression models with telomere length as the outcome in all models. To account for heterogeneity between studies, telomere length was standardised within each study prior to the analysis, using a $\mathrm{z}$-transformation after initially adjusting for age and sex, i.e. scaling the residuals of the regression of telomere length on age and sex to zero mean and unit variance. COPD and asthma cases were coded " 1 ", whereas controls were coded " 0 ". In addition, the spirometric indices were transformed to z-scores taking into account the age, height and sex of each individual. Therefore, GLI reference equations for spirometry [19] were applied to calculate z-scores for FEV1, FVC and FEV1/FVC from skewness (L), the coefficient of variation (S) and the predicted value $(\mathrm{M})$ according to the LMS equations by using the software provided at www.lungfunction.org/tools

Within the linear regression model, we adjusted for ever and current smoking, as well as for study-specific covariates such as case/control status or family structure if appropriate. As both telomere length and lung function measurements were already adjusted for age and sex, the association model itself did not contain these covariates. All analyses were primarily conducted in all available study samples and additionally conducted stratified by sex. The z-transformation of telomere length was performed in males and females separately for the sex-stratified analysis. For the analyses of FEV1, FVC and FEV1/FVC, we additionally stratified into the subgroups "apparently lung healthy", "COPD cases" and "asthmatics".

The group of apparently lung-healthy subjects was determined by exclusion of all subjects with a history of asthma, COPD or other reported respiratory diseases, and all subjects with a reported acute respiratory tract infections within the last 3 weeks. The group was further divided into "current/former" and "never" smokers. In the group of healthy smokers, we further investigated subjects showing the lower and upper $25 \%$ quantiles of lung function (FEV1, FVC or FEV1/FVC). The single-study results were combined in a meta-analysis with inverse variance weighting, performed in the statistical software R (www.r-project.org) using the meta-package with fixed and random effects. As a measure of heterogeneity, inconsistency $\left(\mathrm{I}^{2}\right)$ values were calculated [25] and Cochran's heterogeneity test was performed. As a sensitivity analysis in COPD, we excluded first all subjects $>70$ years of age and then all subjects $>60$ years of age from the analysis.

\section{Results}

COPD

By combining nine studies including 934 COPD cases and 15846 controls according to the GLI criteria (table 1), we observed a significant negative association between COPD and telomere length $(\beta=-0.0982$, $p=0.001$ with fixed effects; $\beta=-0.0953, p=0.012$ with random effects, $I^{2}=27.7 \%$, $p$-value for heterogeneity (phet $)=0.198)$. With COPD defined by the GOLD criteria, there were more COPD cases $(n=1189)$ with an increase in older and a reduction in younger individuals. On the other hand, the association with telomere length using GOLD criteria was more heterogeneous across studies and less significant $(\beta=-0.0676$, $\mathrm{p}=0.018$ with fixed effects; $\beta=-0.0629, \mathrm{p}=0.117$ with random effects; $\mathrm{I}^{2}=42.3 \%$, phet $=0.085$ ). Figure 1 shows study-specific and combined effect estimates of a fixed- and random-effects model with COPD defined by the GLI criteria. The association was strong in the female subsample $(n=10435 ; \beta=-0.1306$, $\mathrm{p}=3.6 \times 10^{-4}$ with fixed effects; $\beta=-0.1312, \mathrm{p}=6.7 \times 10^{-4}$ with random effects; $\mathrm{I}^{2}=5.8 \%$, phet $=0.385$ ) whereas in the male subsample, no significant relationship was detectable $(n=6345 ; \beta=-0.0242, p=0.663$ with fixed effects; $\beta=-0.0329, p=0.622$ with random effects; $\mathrm{I}^{2}=23.5 \%$, phet $=0.250$ ). Online supplementary figure E1 shows the meta-analysis stratified for sex. The two studies showing positive effect estimates, EXATHLETES and NAG-FIN, are selective in their study design: NAG-FIN comprises families of heavy eversmokers, and the EX-ATHLETES are male former elite athletes and matched controls with a mean age of 72.7 years. Since COPD shows an age-related increase in incidence, we conducted sensitivity analyses to investigate whether the observed association is affected by age. Exclusion of subjects $>70$ or $>60$ years of age showed no significant differences for the effect estimates in subgroups of both sexes (fig. E2), confirming that the association is not driven by older age groups.

\section{Asthma}

By combining 13 studies with 2834 asthma cases and 28195 controls (table 2 and fig. 2), we observed a negative association between telomere length and asthma. This was significant in a fixed-effects model $(\beta=-0.0452, p=0.024)$ and suggestive in a random-effects model $\left(\beta=-0.0446, p=0.093 ; I^{2}=30.4 \%\right.$, phet $=0.140)$. The effect was driven by the female subsample $(n=18724, \beta=-0.0764, p=0.002$ with fixed effects; $\beta=-0.0809, \mathrm{p}=0.005$ with random effects; $\mathrm{I}^{2}=15.8 \%$, phet $=0.289$ ) and not seen in the male subsample $(n=12305 ; \beta=0.0182, p=0.594$ with fixed effects; $\beta=0.0139, p=0.771$ with random effects; $\mathrm{I}^{2}=40.3 \%$, phet $=0.080$; fig. E3). 
Study

EX-ATHLETES
FITSA
KORA Age
KORA F3
KORA F4
NAG-FIN
NFBC 1966
TwinGene
TwinsUK

Fixed-effects model Random-effects model

Females

Males
Total $\mathbf{n}$ Cases $\mathbf{n}$

577
386
905
875
2803
1718
4984
597
3935



43
22
54
36
243
126
147
52
211

\section{1}

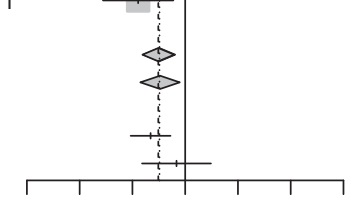

$\begin{array}{lllll}-0.6-0.4-0.2 & 0 & 0.2 & 0.4 & 0.6\end{array}$

$\beta$

FIGURE 1 Associations between telomere length and chronic obstructive pulmonary disease (COPD). Forest plot comparing effects between studies, and showing combined effects in a fixed-effects model as well as in a random-effects model. Sex-stratified results are based on random-effects models. 95\% confidence intervals are given for all estimates. COPD was defined by the Global Lungs Initiative criteria. FITSA: Finnish Twin Study on Ageing; KORA: Cooperative Health Research in the Region Augsburg; NAG-FIN: Nicotine Addiction Genetics Finland; NFBC: Northern Finland Birth Cohort Study.

\section{Lung function}

By combining the data of seven studies, totalling 12595 individuals (table 3 and fig. 3), we observed highly significant positive associations between telomere length and spirometric indices measured as FEV1 $\left(\beta=0.0455, p=1.07 \times 10^{-7}\right.$ with fixed and random effects; $\left.I^{2}=0 \%\right)$, FVC $\left(\beta=0.0401, p=2.07 \times 10^{-5}\right.$ with fixed and random effects; $\left.\mathrm{I}^{2}=0 \%\right)$ and $\mathrm{FEV} 1 / \mathrm{FVC}\left(\beta=0.0238, \mathrm{p}=5.27 \times 10^{-3}\right.$ with fixed and random effects; $\left.\mathrm{I}^{2}=0 \%\right)$. The effect for FEV1 was higher in females than in males $(\beta=0.0548$ versus $\beta=0.286$ with random effects). The meta-analysis stratified by sex is shown in figure E4.

Figure 4 shows the association results stratified by lung health status. Compared with "healthy" controls, we found somewhat stronger effects in the disease subgroups of COPD and asthma cases. Comparisons of the effect estimates stratified by health status and by sex are presented in figure E5. The stronger effects found in COPD were predominantly seen in males and were less obvious in females. The opposite was observed for asthma, where the effects were strong in females. With regard to smoking, within the healthy subgroups, the

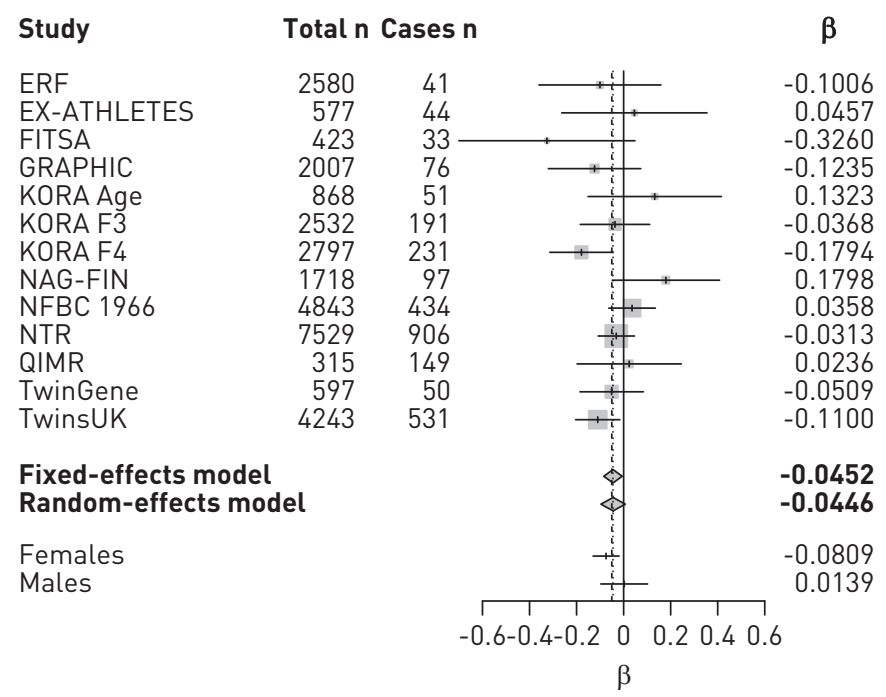

FIGURE 2 Association between telomere length and asthma. Forest plot comparing effects between studies, and showing combined effects in a fixed-effects model as well as in a random-effects model. Sex-stratified results are based on randomeffects models. 95\% confidence intervals are given for all estimates. ERF: Erasmus Rucphen Family; FITSA: Finnish Twin Study on Ageing; KORA: Cooperative Health Research in the Region Augsburg; NAG-FIN: Nicotine Addiction Genetics Finland; NFBC: Northern Finland Birth Cohort Study; NTR: Netherlands Twin Register; QIMR: Queensland Institute of Medical Research. 
TABLE 3 Characteristics of studies in lung function index analysis

\begin{tabular}{|c|c|c|c|c|c|c|c|}
\hline Study & FITSA & KORA Age & KORA F3 & KORA F4 & NFBC 1966 & TwinFat & TwinsUK \\
\hline Subjects & 386 & 905 & 875 & 1291 & 4984 & 219 & 3935 \\
\hline Females n (\%) & $386(100)$ & $451(49.8)$ & 458 (52.3) & $691(53.5)$ & 2594 (52.0) & $101(46.1)$ & 3687 (93.7) \\
\hline FEV1 L & $2.20 \pm 0.49$ & $2.49 \pm 0.72$ & $3.56 \pm 0.82$ & $3.34 \pm 0.82$ & $3.95 \pm 0.79$ & $4.03 \pm 0.84$ & $2.74 \pm 0.66$ \\
\hline FVC L & $2.82 \pm 0.59$ & $3.36 \pm 0.93$ & $4.3 \pm 1.02$ & $4.30 \pm 1.01$ & $4.72 \pm 0.99$ & $4.74 \pm 1.12^{\#}$ & $3.41 \pm 0.73$ \\
\hline FEV1/FVC \% & $78.19 \pm 8.29$ & $74.32 \pm 8.42$ & $83.09 \pm 7.08$ & $77.65 \pm 6.16$ & $84.11 \pm 6.43$ & $84.98 \pm 6.07^{\#}$ & $80.42 \pm 8.47$ \\
\hline FEV1/FVC z-score & $-0.54 \pm 1.13$ & $-0.26 \pm 0.97$ & $0.42 \pm 1.13$ & $-0.28 \pm 0.89$ & $0.15 \pm 1.13$ & $0.20 \pm 0.96 \#$ & $-0.02 \pm 1.12$ \\
\hline $\begin{array}{l}\text { Smoking status never/ } \\
\text { former/current }\end{array}$ & $333 / 31 / 21$ & $510 / 351 / 44$ & $377 / 260 / 238$ & $496 / 496 / 299$ & $2479 / 592 / 1913$ & $110 / 54 / 55$ & $2760 / 743 / 432$ \\
\hline
\end{tabular}

Data are presented as $\mathrm{n}$ or mean $\pm \mathrm{SD}$, unless otherwise stated. FITSA: Finnish Twin Study on Ageing; KORA: Cooperative Health Research in the Region Augsburg; NFBC: Northern Finland Birth Cohort Study; FEV1: forced expiratory volume in $1 \mathrm{~s} ;$ FVC: forced vital capacity. ${ }^{\#}: \mathrm{n}=124$.

observed effects were stronger in "healthy never-smokers" than in "healthy ever-smokers" for FEV1 and FVC (fig. E6). To further explore the contribution of smoking, we stratified the group of healthy smokers (current or ex-smokers) by lung function status (lower $25 \%$ or upper $25 \%$ within this subgroup). The results suggest that there was no difference between those two groups for FEV1. In FVC, an effect was only seen in individuals with poor lung function (lower 25\%). Although differences between subgroups were noticeable, they did not differ significantly.

\section{Discussion}

We conducted a large study investigating the relationship between telomere length and lung function and disease, assessed in multiple European cohorts unselected for lung disease. We found highly significant positive associations between telomere length from peripheral leukocytes and spirometric indices measured as FEV1, FVC and FEV1/FVC in a meta-analysis of seven studies with 12595 individuals. When stratifying by lung health status, larger effects were seen in the subgroups of COPD patients or asthmatics. This suggests that lung function decline, in part, reflects biological ageing due to intrinsic processes, which are likely to be aggravated in asthma or COPD due to mechanisms promoting cell senescence.

Tobacco smoke is a well-known environmental factor that promotes ageing processes and cellular senescence [8]. The recent large, population-based study by RoDE et al. [18] revealed that having ever smoked and the cumulative pack-years smoked were associated with telomere length in 46396 adults. This is in line with previous population-based findings [26]. However, in clinical studies, these effects were not seen $[14,17,20]$. When stratifying by smoking status, we observed no evident associations between telomere
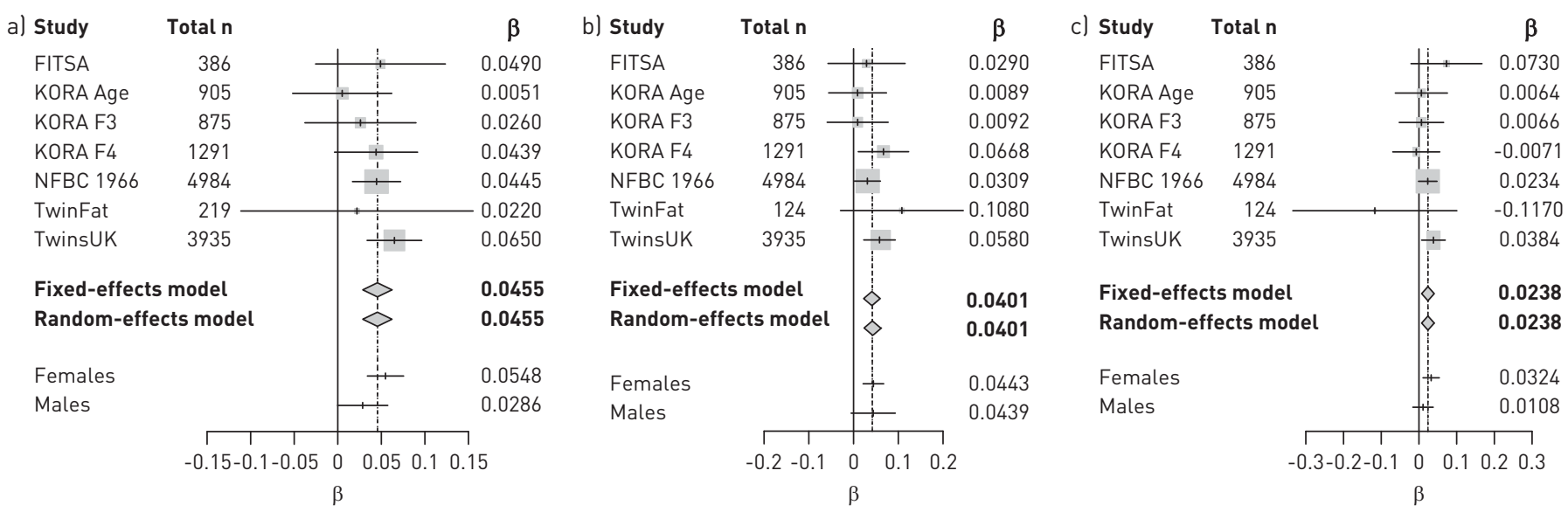

FIGURE 3 Telomere length is associated with a) forced expiratory volume in $1 \mathrm{~s}$ (FEV1), b) forced vital capacity (FVC) and c) FEV1/FVC. Forest plots comparing effects between studies, and showing combined effects in fixed-effects models as well as in random-effects models. Sex-stratified results are based on randomeffects models. 95\% confidence intervals are given for all estimates. FITSA: Finnish Twin Study on Ageing; KORA: Cooperative Health Research in the Region Augsburg; NFBC: Northern Finland Birth Cohort Study. 
a)

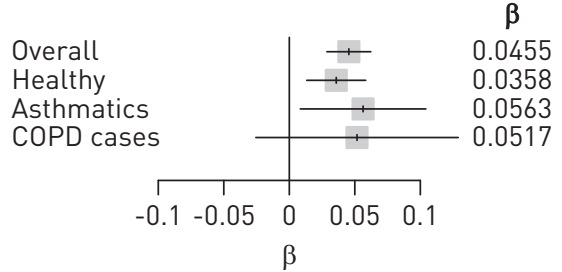

b)

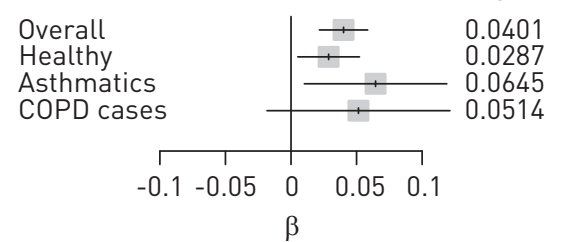

c)

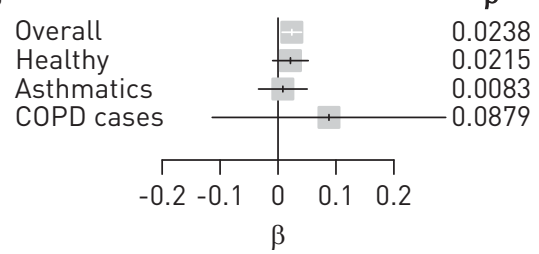

FIGURE 4 Associations between telomere length and a) forced expiratory volume in $1 \mathrm{~s}$ (FEV1), b) forced vital capacity (FVC) and c) FEV1/FVC, stratified by health status. Forest plots showing effects in the overall sample as well as in "apparently lung-healthy" individuals, asthmatics and patients with chronic obstructive pulmonary disease (COPD) only to test the impact of lung disease on the effect. Effects are based on random-effects models. 95\% confidence intervals are given for all estimates.

length and lung function indices in ever-smokers. In addition, stratifying by lung function within the group of healthy smokers (current and ex-smokers) did not clarify the issue, although for FVC, an effect could be detected in smokers of the lower quartile. The underlying reason for not detecting an effect may be found in the complex interactions between smoking and genetic and/or lifestyle factors, such as dietary antioxidants or physical activity, affecting biological age and its markers [1,3,27-32], but also in the heterogeneity of smoking exposure, and its amount and duration.

Up to now, a limited number of studies have addressed the association between lung function and telomere length. MUi et al. [20] observed a positive association in a sample of COPD patients and Tsuj et al. [16] reported that airflow limitation and alveolar cell senescence were positively correlated with each other. Other studies failed to find significant associations between spirometric parameters and telomere length $[14,17,33]$, although one study found that parameters of gas exchange (arterial oxygen and carbon dioxide pressures) were associated with telomere length in COPD patients [14]. The large population-based study by RoDE et al. [18] found weak but statistically significant positive correlations between telomere length and spirometric indices, which is confirmed by our results. Furthermore, we observed the effects to be somewhat stronger in COPD or asthma patients compared with healthy individuals, suggesting that intrinsic agerelated processes may be aggravated by chronic inflammatory processes occurring either locally in the lung or systemically.

In both COPD and asthma patients, we observed shorter telomeres compared with healthy controls. In COPD, the association was stronger when defining cases by the age-dependent LLN for FEV1/FVC according to the GLI criteria [19] compared with the fixed cut-off given by GOLD [21]. This suggests that the GLI approach better suits the transition from health to disease. For COPD and asthma, effect estimates appeared to be larger in females than in males, although sex differences were not statistically significant. With respect to COPD, this might be consistent with the observation that females appear to be more susceptible to COPD than males [34] but the causality certainly needs to be proved. Whereas our findings for asthma are novel, the results for COPD are in line with previous findings in case-control [14-17] and population-based studies [18]. This suggests that, as already shown for other chronic diseases [1, 3, 35], accelerated ageing may contribute to the pathogenesis of asthma and COPD.

Our epidemiological finding is supported by results from clinical studies showing shorter telomeres in alveolar epithelial and endothelial cells from patients with emphysema [16]. AMSELLEM et al. [15] reported a higher percentage of pulmonary vascular endothelial cells stained for senescence markers in COPD patients than in lung healthy controls. This is in line with senescent-type growth characteristics of lung fibroblasts [36]. Furthermore, cellular senescence of pulmonary smooth muscle cells appears to be involved in the pathogenesis of pulmonary hypertension in COPD [37]. Several mechanisms have been proposed for abnormal ageing in COPD, including chronic inflammation, oxidative stress, mitochondrial dysfunction, altered energy metabolism, abnormal regulation of ageing processes, cellular and immunosenescence, and telomere dysfunction $[3,7-9,15]$. Analogous mechanisms in asthma have not been explored in detail but, due to the inflammatory nature of both diseases, some of the aforementioned factors are also likely to play a role in asthma. Possible consequences for both diseases are very difficult to predict but one might speculate that the ageing processes may be reflected in shorter life expectancy and an increased risk of cognitive impairment and dementia as reported for asthma and COPD [38-40].

The observed associations between telomere length and both diseases were not as strong as one might have initially expected, considering the large sample size. In the case of COPD, in the population-based approach of Rode et al. [18], a much weaker association was also observed than previous case-control studies had suggested [14]. In our case, this may be related to the fact that mostly mild-to-moderate disease stages were included in the study samples. For example, for COPD, in the KORA cohorts, $94.3 \%$ of COPD cases were in 
GOLD stages I and II. RoDE et al. [18] also observed weaker effects for stages I (OR 1.09, 95\% CI 0.97-1.23) and II (OR 1.17, 95\% CI 1.04-1.31) than for more advanced GOLD, stages after multiple adjustment. In the case of asthma, the weakness of association could be due to the different methods of ascertaining asthma cases or phenotypic heterogeneity.

Moreover, as in many other studies, telomere length was measured in circulating leukocytes and not in the cells of the primarily affected organ, i.e. the lung. Studies addressing the comparability of telomere length between different tissues found strong correlations in healthy individuals, but somewhat blunted ones in disease [41-44]. We cannot rule out that this may be a factor that affects the association of telomere length with lung diseases. Leukocyte telomere length is a complex trait that is shaped by many factors including genetic, epigenetic, lifestyle and environmental determinants, and their complex interaction is hardly understood. In the present analysis, we therefore adjusted only for well accepted determinants. Certainly, the population-based epidemiological nature of the studies included comes along with distinct limitations. Population-based samples typically only scarcely include severe cases of lung diseases; therefore, early disease stages predominate, in which age-related mechanisms may not be as significant as in more advanced stages that are primarily included in clinical cohorts [14-17]. Furthermore, our study design did not allow us to take into account the known heterogeneity of COPD and asthma phenotypes. These entities may have different impacts on telomere length or may trigger premature ageing processes via telomere-independent mechanisms, as reported for fibroblasts of patients with lung emphysema [45]. Unlike in clinical studies, diagnosis of COPD and asthma had to rely mainly on self-reported physician diagnosis, which certainly adds some potential for misclassification error. However, we employed the recent GLI reference values [19] for COPD diagnosis wherever possible. In addition, confounding effects, such as smoking or medication, could not be accounted for in the same way as in a matched case-control study design.

As this analysis combines a number of studies that are heterogeneous in their design (online supplementary material), we report results of random-effects models in addition to the results based on fixed effects, in order to account for this heterogeneity. Naturally, confidence intervals are larger with random effects. While both models reach statistical significance in COPD, statistical significance was only reached with the fixedeffects model for asthma. The observed effect point estimates, however, were comparable between the fixedand the random-effects model, supporting the biological significance of our findings. In COPD, our sensitivity analysis did not show an age dependency (fig. E2). Two studies showing positive effect estimates for COPD were selective in their study design; in the case of the EX-ATHLETES study, the positive effect of lifetime physical exercise on biological age may have played a role [3, 27, 46, 47].

\section{Conclusions}

We investigated the relationship of telomere length, as a marker of biological age, with lung function and respiratory disease. We found highly significant positive associations between telomere length and lung function. Furthermore, shorter telomeres were seen in patients with COPD and asthma cases compared with healthy controls. Our results provide indirect evidence for the hypothesis that premature ageing is involved in the pathophysiology of COPD and asthma, and that lung function partially reflects biological ageing due to intrinsic processes. These processes are likely to be aggravated by lung diseases promoting cellular senescence, although the average effect seems to be not particularly strong.

\section{Acknowledgements}

A detailed list of acknowledgements is provided in the online supplementary material.

The authors' affiliations are: E. Albrecht, Institute of Genetic Epidemiology, Helmholtz Zentrum München - German Research Center for Environmental Health, Neuherberg, Germany; E. Sillanpää, Gerontology Research Center and Dept of Health Sciences, University of Jyväskylä, Jyväskylä, Finland; S. Karrasch, Institute and Outpatient Clinic for Occupational, Social and Environmental Medicine, Ludwig-Maximilians-Universität, and Institute of General Practice, University Hospital Klinikum rechts der Isar, Technische Universität München, Munich, and Institute of Epidemiology I, Helmholtz Zentrum München - German Research Center for Environmental Health, Neuherberg, Germany; A.C. Alves, Dept of Epidemiology and Biostatistics, MRC-HPA (Health Protection Agency) Centre for Environment and Health, School of Public Health, Faculty of Medicine, Imperial College, London, UK; V. Codd, Dept of Cardiovascular Sciences, University of Leicester, and Leicester NIHR Biomedical Research Unit in Cardiovascular Disease, Glenfield Hospital, Leicester, UK; I. Hovatta, Mental Health and Substance Abuse Services, National Institute for Health and Welfare, and Dept of Biosciences, Viikki Biocentre, University of Helsinki, Helsinki, Finland; J.L. Buxton, Section of Investigative Medicine, Dept of Medicine, Imperial College London, London, UK; C.P. Nelson, Dept of Cardiovascular Sciences, University of Leicester, and Leicester NIHR Biomedical Research Unit in Cardiovascular Disease, Glenfield Hospital, Leicester, UK; L. Broer, Netherlands Consortium for Healthy Aging, Leiden University Medical Center, Leiden, and Dept of Epidemiology, Erasmus Medical Center, Rotterdam, The Netherlands; S. Hägg, Dept of Medical Epidemiology and Biostatistics, Karolinska Institutet, Stockholm, and Dept of Medical Sciences, Molecular Epidemiology and Science for Life Laboratory, Uppsala University, Uppsala, Sweden; M. Mangino, Dept of Twin Research and Genetic Epidemiology, King's College London, London, UK; G. Willemsen, Dept of Biological Psychology, VU University Amsterdam, Amsterdam, The Netherlands; I. Surakka, Public Health Genomics Unit, Dept of Chronic Disease Prevention, National 
Institute for Health and Welfare, and Institute for Molecular Medicine Finland FIMM, University of Helsinki, Helsinki, Finland; M.A.R. Ferreira, Queensland Institute of Medical Research, Brisbane, QLD, Australia; N. Amin, Dept of Epidemiology, Erasmus Medical Center, Rotterdam, The Netherlands; B.A. Oostra, Dept of Clinical Genetics, Erasmus Medical Center, Rotterdam, The Netherlands; H.M. Bäckmand, University of Helsinki, Hjelt Institute, Dept of Public Health, Helsinki, and Health and Social Welfare Dept, Vantaa, Finland; M. Peltonen, Dept of Chronic Disease Prevention, National Institute for Health and Welfare, Helsinki, Finland; S. Sarna, University of Helsinki, Hjelt Institute, Dept of Public Health, Helsinki, Finland; T. Rantanen, Gerontology Research Center and Dept of Health Sciences, University of Jyväskylä, Jyväskylä, Finland; S. Sipilä, Gerontology Research Center and Dept of Health Sciences, University of Jyväskylä, Jyväskylä, Finland; T. Korhonen, University of Helsinki, Hjelt Institute, Dept of Public Health, Helsinki, Finland; P.A.F. Madden, Washington University School of Medicine, St Louis, MO, USA; C. Gieger, Institute of Genetic Epidemiology, Helmholtz Zentrum München - German Research Center for Environmental Health, Neuherberg, Germany; R.A. Jörres, Institute and Outpatient Clinic for Occupational, Social and Environmental Medicine, Ludwig-Maximilians-Universität, and Comprehensive Pneumology Center Munich (CPC-M), Member of the German Center for Lung Research, Munich, Germany; J. Heinrich, Institute of Epidemiology I, Helmholtz Zentrum München - German Research Center for Environmental Health, Neuherberg, and CPC-M, Member of the German Center for Lung Research, Munich, Germany; J. Behr, CPC-M, Member of the German Center for Lung Research, and Dept of Internal Medicine V, University of Munich, Munich, Germany; R.M. Huber, CPC-M, Member of the German Center for Lung Research, and Dept of Internal Medicine V, and Thoracic Oncology Centre Munich, University of Munich, Munich, Germany; A. Peters, Research Unit of Molecular Epidemiology, Helmholtz Zentrum München - German Research Center for Environmental Health, Institute of Epidemiology II, Helmholtz Zentrum München - German Research Center for Environmental Health, Neuherberg, and Munich Heart Alliance, Munich, Germany; K. Strauch, Institute of Genetic Epidemiology, Helmholtz Zentrum München - German Research Center for Environmental Health, Neuherberg, and Institute of Medical Informatics, Biometry and Epidemiology, Ludwig-Maximilians-Universität, Munich, Germany; H.E. Wichmann, Institute of Epidemiology I, Helmholtz Zentrum München - German Research Center for Environmental Health, Neuherberg, Institute of Medical Informatics, Biometry and Epidemiology, Ludwig-Maximilians-Universität, and Klinikum Grosshadern, Munich, Germany; M. Waldenberger, Research Unit of Molecular Epidemiology, Helmholtz Zentrum München - German Research Center for Environmental Health, Neuherberg, Germany; A.I.F. Blakemore, Section of Investigative Medicine, Dept of Medicine, Imperial College London, London, UK; E.J.C. de Geus, Dept of Biological Psychology, VU University Amsterdam, Amsterdam, The Netherlands; D.R. Nyholt, Queensland Institute of Medical Research, Brisbane, QLD, Australia; A.K. Henders, Queensland Institute of Medical Research, Brisbane, QLD, Australia; P.L. Piirilä, Unit of Clinical Physiology, HUS Medical Imaging Center, Helsinki University Hospital, Helsinki, Finland; A. Rissanen, Obesity Research Unit, Dept of Psychiatry, Helsinki University Central Hospital, Helsinki, Finland; P.K.E. Magnusson, Dept of Medical Epidemiology and Biostatistics, Karolinska Institutet, Stockholm, Sweden; A. Viñuela, Dept of Twin Research and Genetic Epidemiology, King's College London, London, UK; K.H. Pietiläinen, Institute for Molecular Medicine Finland FIMM, University of Helsinki, and Obesity Research Unit, Dept of Medicine, Division of Endocrinology, Helsinki University Central Hospital and University of Helsinki, Helsinki, Finland; N.G. Martin, Queensland Institute of Medical Research, Brisbane, QLD, Australia; N.L. Pedersen, Dept of Medical Epidemiology and Biostatistics, Karolinska Institutet, Stockholm, Sweden; D.I. Boomsma, Dept of Biological Psychology, VU University Amsterdam, Amsterdam, The Netherlands; T.D. Spector, Dept of Twin Research and Genetic Epidemiology, King's College London, London, UK; C.M. van Duijn, Netherlands Consortium for Healthy Aging, Leiden University Medical Center, Leiden, and Dept of Epidemiology, Erasmus Medical Center, Rotterdam, The Netherlands; J. Kaprio, Mental Health and Substance Abuse Services, National Institute for Health and Welfare, Institute for Molecular Medicine Finland FIMM, University of Helsinki, and University of Helsinki, Hjelt Institute, Dept of Public Health, Helsinki, Finland; N.J. Samani, Dept of Cardiovascular Sciences, University of Leicester, and Leicester NIHR Biomedical Research Unit in Cardiovascular Disease, Glenfield Hospital, Leicester, UK; M-R. Jarvelin, Dept of Epidemiology and Biostatistics, MRC-HPA Centre for Environment and Health, School of Public Health, Faculty of Medicine, Imperial College, London, UK, Institute of Health Sciences and Biocenter Oulu, University of Oulu, Unit of General Practice, University Hospital Oulu, and Dept of Lifecourse and Services, National Institute for Health and Welfare, Oulu, Finland; H. Schulz, Institute of Epidemiology I, Helmholtz Zentrum München - German Research Center for Environmental Health, Neuherberg Germany.

\section{References}

Armanios M, Blackburn EH. The telomere syndromes. Nat Rev Genet 2012; 13: 693-704.

Calado R, Young N. Telomeres in disease. F1000 Med Rep 2012; 4: 8.

Zhu H, Belcher M, van der Harst P. Healthy aging and disease: role for telomere biology? Clin Sci (Lond) 2011; 120: $427-440$.

4 Armanios M. Telomeres and age-related disease: how telomere biology informs clinical paradigms. J Clin Invest 2013; 123: 996-1002.

Dyer C. The interaction of ageing and lung disease. Chron Respir Dis 2012; 9: 63-67.

Dyer CA, Stockley RA. The aging lung. Rev Clin Gerontol 2006; 16: 99-111.

Ito K, Barnes PJ. COPD as a disease of accelerated lung aging. Chest 2009; 135: 173-180.

Karrasch S, Holz O, Jörres RA. Aging and induced senescence as factors in the pathogenesis of lung emphysema. Respir Med 2008; 102: 1215-1230.

9 Faner R, Rojas M, Macnee W, et al. Abnormal lung aging in chronic obstructive pulmonary disease and idiopathic pulmonary fibrosis. Am J Respir Crit Care Med 2012; 186: 306-313.

10 Moyzis RK, Buckingham JM, Cram LS, et al. A highly conserved repetitive DNA sequence, (TTAGGG) $n$, present at the telomeres of human chromosomes. Proc Natl Acad Sci USA 1988; 85: 6622-6626.

11 Harley CB, Futcher AB, Greider CW. Telomeres shorten during ageing of human fibroblasts. Nature 1990; 345: 458-460.

12 Broer L, Codd V, Nyholt DR, et al. Meta-analysis of telomere length in 19,713 subjects reveals high heritability, stronger maternal inheritance and a paternal age effect. Eur J Hum Genet 2013; 21: 1163-1168.

13 Lee J, Sandford AJ, Connett JE, et al. The relationship between telomere length and mortality in chronic obstructive pulmonary disease (COPD). PLoS One 2012; 7: e35567. 
Savale L, Chaouat A, Bastuji-Garin S, et al. Shortened telomeres in circulating leukocytes of patients with chronic obstructive pulmonary disease. Am J Respir Crit Care Med 2009; 179: 566-571.

15 Amsellem V, Gary-Bobo G, Marcos E, et al. Telomere dysfunction causes sustained inflammation in chronic obstructive pulmonary disease. Am J Respir Crit Care Med 2011; 184: 1358-1366.

16 Tsuji T, Aoshiba K, Nagai A. Alveolar cell senescence in patients with pulmonary emphysema. Am J Respir Crit Care Med 2006; 174: 886-893.

17 Houben JMJ, Mercken EM, Ketelslegers HB, et al. Telomere shortening in chronic obstructive pulmonary disease. Respir Med 2009; 103: 230-236.

18 Rode L, Bojesen SE, Weischer M, et al. Short telomere length, lung function and chronic obstructive pulmonary disease in 46,396 individuals. Thorax 2013; 68: 429-435.

19 Quanjer PH, Stanojevic S, Cole TJ, et al. Multi-ethnic reference values for spirometry for the 3-95-yr age range: the global lung function 2012 equations. Eur Respir J 2012; 40: 1324-1343.

20 Mui TSY, Man JM, McElhaney JE, et al. Telomere length and chronic obstructive pulmonary disease: evidence of accelerated aging. J Am Geriatr Soc 2009; 57: 2372-2374.

21 Rabe KF, Hurd S, Anzueto A, et al. Global strategy for the diagnosis, management, and prevention of chronic obstructive pulmonary disease: GOLD executive summary. Am J Respir Crit Care Med 2007; 176: 532-555.

22 Miller MR, Hankinson J, Brusasco V, et al. Standardisation of spirometry. Eur Respir J 2005; 26: 319-338.

23 Quanjer PH, Tammeling GJ, Cotes JE, et al. Lung volumes and forced ventilatory flows. Eur Respir J 1993; 6: Suppl. 16, 5-40.

24 Cawthon RM. Telomere measurement by quantitative PCR. Nucleic Acids Res 2002; 30 : e47.

25 Higgins JPT, Thompson SG, Deeks JJ, et al. Measuring inconsistency in meta-analyses. BMJ 2003; 327: 557-560.

26 Babizhayev MA, Yegorov YE. Smoking and health: association between telomere length and factors impacting on human disease, quality of life and life span in a large population-based cohort under the effect of smoking duration. Fundam Clin Pharmacol 2011; 25: 425-442.

27 Sun Q, Shi L, Prescott J, et al. Healthy lifestyle and leukocyte telomere length in U.S. women. PLoS One 2012; 7: e38374.

28 Pellatt AJ, Wolff RK, Lundgreen A, et al. Genetic and lifestyle influence on telomere length and subsequent risk of colon cancer in a case control study. Int J Mol Epidemiol Genet 2012; 3: 184-194.

29 Mirabello L, Huang WY, Wong JY, et al. The association between leukocyte telomere length and cigarette smoking, dietary and physical variables, and risk of prostate cancer. Aging Cell 2009; 8: 405-413.

30 Yen YC, Lung FW. Older adults with higher income or marriage have longer telomeres. Age Ageing 2013; 42: 234-239.

31 Jackowska M, Hamer M, Carvalho LA, et al. Short sleep duration is associated with shorter telomere length in healthy men: findings from the Whitehall II cohort study. PLoS One 2012; 7: e47292.

32 Bentley AR, Kritchevsky SB, Harris TB, et al. Dietary antioxidants and forced expiratory volume in $1 \mathrm{~s}$ decline: the Health, Aging and Body Composition study. Eur Respir J 2012; 39: 979-984.

33 Mather KA, Jorm AF, Milburn PJ, et al. No associations between telomere length and age-sensitive indicators of physical function in mid and later life. J Gerontol A Biol Sci Med Sci 2010; 65: 792-799.

34 Aryal S, Diaz-Guzman E, Mannino DM. COPD and gender differences: an update. Transl Res 2013; 162: $208-218$.

35 El Assar M, Angulo J, Vallejo S, et al. Mechanisms involved in the aging-induced vascular dysfunction. Front Physiol 2012; 3: 132 .

36 Holz O, Zuhlke I, Jaksztat E, et al. Lung fibroblasts from patients with emphysema show a reduced proliferation rate in culture. Eur Respir J 2004; 24: 575-579.

37 Noureddine H, Gary-Bobo G, Alifano M, et al. Pulmonary artery smooth muscle cell senescence is a pathogenic mechanism for pulmonary hypertension in chronic lung disease. Circ Res 2011; 109: 543-553.

38 Jia H, Zack MM, Thompson WW. The effects of diabetes, hypertension, asthma, heart disease, and stroke on quality-adjusted life expectancy. Value Health 2013; 16: 140-147.

39 Murray CJ, Richards MA, Newton JN, et al. UK health performance: findings of the Global Burden of Disease Study 2010. Lancet 2013; 381: 997-1020.

40 Rusanen M, Ngandu T, Laatikainen T, et al. Chronic obstructive pulmonary disease and asthma and the risk of mild cognitive impairment and dementia: a population based CAIDE study. Curr Alzheimer Res 2013; 10: 549-555.

41 Butler MG, Tilburt J, DeVries A, et al. Comparison of chromosome telomere integrity in multiple tissues from subjects at different ages. Cancer Genet Cytogenet 1998; 105: 138-144.

42 Friedrich U, Griese E, Schwab M, et al. Telomere length in different tissues of elderly patients. Mech Ageing Dev 2000; 119: 89-99.

43 Zhang W, Chen Y, Wang Y, et al. Short telomere length in blood leucocytes contributes to the presence of atherothrombotic stroke and haemorrhagic stroke and risk of post-stroke death. Clin Sci (Lond) 2013; 125: 27-36.

44 Wong LS, Huzen J, de Boer RA, et al. Telomere length of circulating leukocyte subpopulations and buccal cells in patients with ischemic heart failure and their offspring. PLoS One 2011; 6: e23118.

45 Muller KC, Welker L, Paasch K, et al. Lung fibroblasts from patients with emphysema show markers of senescence in vitro. Respir Res 2006; 7: 32

46 Trappe S, Hayes E, Galpin A, et al. New records in aerobic power among octogenarian lifelong endurance athletes. J Appl Physiol 2013; 114: 3-10.

47 Joyner MJ, Barnes JN. I am 80 going on 18: exercise and the fountain of youth. J Appl Physiol 2013; 114: 1-2. 